\title{
Na-montmorillonite modified with ammonium salts and azobenzene as a photoactive nanomaterial
}

\author{
Anna Koteja, Jakub Matusik \\ AGH University of Science and Technology, Faculty of Geology, Geophysics and Environmental Protection, \\ Department of Mineralogy, Petrography and Geochemistry; al. A. Mickiewicza 30, 30-059 Krakow, Poland; \\ e-mail: jakub_matusik@wp.pl
}

(C) 2016 Authors. This is an open access publication, which can be used, distributed and reproduced in any medium according to the Creative Commons CC-BY 4.0 License requiring that the original work has been properly cited.

Modification of inorganic solid structures (e.g. minerals) with organic molecules is a constantly developed topic in material sciences. The organic functionalization leads to the production of new materials with integrated properties of both the organic and inorganic component. In the presented study we have modified a Na-montmorillonite with alkylammonium surfactants and subsequently azobenzene, in order to obtain a nanomaterial that shows response to UV radiation. Azobenzene is a photoswitchable organic molecule capable to change its conformation upon UV radiation from the trans- to cis-azobenzene isomer. This reaction is coupled with a change of the molecules shape and dimensions (Klajn 2010). The montmorillonite is a layered aluminosilicate that serves as an excellent host structure for organic guest species. Due to the net negative layer charge it shows the ability to swell and to exchange the originally present interlayer cations. These properties allow the intercalation of bulk organic molecules and to control their arrangement. Much attention has been paid to the possibility of transferring the photoswitching ability of organic molecule into the motion of the whole organo-mineral structure (Heinz et al. 2008). Such nanoswitch is particularly appealing as it is controlled with radiation - remotely and at a precise location. The efficiency of a synthesized nanoswitch depends on an accurate selection of the host and guest component. The target of this study to test a series of organic surfactants and to establish a modification pathway that leads to obtaining a material most promising in the view of its photoresponsive behavior.

The montmorillonite modification was performed in a two-step procedure, as the direct intercalation of a nonionic azobenzene is not possible. First, the Na-montmorillonite (denoted SWy) was ion-exchanged with trimethylalkylammonium cations abbreviated $\mathrm{C}_{n}$ and benzyldimethylalkylammonium cations $-\mathrm{BC}_{n}$, where $\mathrm{n}$ refers to the number of carbon atoms in the alkyl chain and is equal to 12,14 or 16 . In the second step the organo-montmorillonites were reacted with azobenzene $(\mathrm{AzBz})$ for $24 \mathrm{~h}$ at $120^{\circ} \mathrm{C}$ in a hermetically closed teflon vessel. The yellowish products were characterized with the X-Ray diffraction (XRD), the infrared spectroscopy (FTIR) and CHN elemental analysis.

In all cases the intercalation of the ammonium cation caused an increase of the montmorillonites basal spacing $\left(d_{001}\right)$. The $d_{001}$ values were equal to $16.4 \AA, 18.2 \AA$ and $20.5 \AA$ for $S W y-C_{12}, S W y-C_{14}$ and $\mathrm{SWy}-\mathrm{C}_{16}$, respectively. The samples modified with the $\mathrm{BC}_{n}$ cations showed $\sim 1.5 \AA$ larger basal spacing, due to the presence of the benzyl group in the intercalated molecule. A linear relationship was observed between the $d_{001}$ value and the alkyl chain length of the introduced salts. This suggests that the organic cations formed paraffin-type aggregates in the interlayer (Ogawa et al. 1999) where the molecules are inclined to the layer surface. The FTIR spectra of modified SWy sample 
showed intense bands corresponding to $\mathrm{CH}_{2}$ vibration modes. Along with the increasing alkyl chain length the $\mathrm{CH}_{2}$ stretching bands shifted towards lower energies. This is an effect of growing packing density of alkylammonium molecules in the interlayer (He et al. 2004) and it is coupled with straightening of the alkyl chains due to transformation of disordered gauche conformer to the ordered all-trans conformer (Vaia et al. 1994). It can be concluded that the longer alkyl chains $\left(\mathrm{C}_{16}\right.$ and $\left.\mathrm{BC}_{16}\right)$ form more ordered, solid-like aggregates in the interlayer space. The molar content of organic molecules was calculated basing on the CHN elemental analysis. The amount of intercalated alkylammonium cations was nearly equal to the cation exchange capacity (CEC) of montmorillonite $-88.9 \mathrm{meq} / 100 \mathrm{~g}$.

The reaction with azobenzene was most effective for montmorillonite modified with the alkylammonium cations having the longest chains as confirmed by the XRD patterns. The $d_{001}$ values of $\mathrm{SWy}_{16} \mathrm{C}_{16}$ and $\mathrm{SWy}_{\mathrm{B}} \mathrm{BC}_{16}$ samples after reaction with AzBz increased to $36.9 \AA$ and $35.9 \AA$, respectively. Well resolved and intense (001) peaks as well as the presence of the $2^{\text {nd }}$ and $3^{\text {rd }}$ order reflections indicated a highly ordered structure of these intercalates. On the contrary, diffraction peaks were less resolved and broadened for samples prepared with the shorter $\mathrm{C}_{12}, \mathrm{C}_{14}, \mathrm{BC}_{12}$ and $\mathrm{BC}_{14}$ molecules after reaction with AzBz. Based on these results, it is assumed that the long chain alkylammonium ions are more effective surfactants for the further intercalation of azobenzene into the montmorillonites interlayer space. The obtained highly ordered structures are promising materials for application as photo-actuated nanoswitches.

This project was funded by the National Science Centre, Poland under research project no. $2014 / 13 /$ B/ST10/01326.

\section{REFERENCES}

He H., Frost R.L. \& Zhu J., 2004. Infrared study of HDT$\mathrm{MA}^{+}$intercalated montmorillonite. Spectrochimica Acta Part A: Molecular and Biomolecular Spectroscopy, 60, 2853-2859.

Heinz H., Vaia R.A., Koermer H. \& Farmer B.I., 2008. Photoisomerization of Azobenzene Grafted to Layered Silicates: Simulation and Experimental Challenges. Chemistry of Materials, 20, 6444-6456.

Klajn R., 2010. Immobilized azobenzenes for the construction of photoresponsive materials. Pure and Applied Chemistry, 82, 2247-2279.

Ogawa M., Hama M. \& Kuroda K., 1999. Photochromism of azobenzene in the hydrophobic interlayer spaces of dialkyldimethylammonium-fluor-tetrasilicic mica films. Clay Minerals, 34, 213-220.

Vaia R.A., Teukolsky R.K. \& Giannelis E.P., 1994. Interlayer Structure and Molecular Environment of Alkylammonium Layered Silicates. Chemistry of Materials, 6, 1017-1022. 